\title{
Funcionamiento sexual y personalidad: análisis diferencial en función del género
}

\author{
Livia García Pérez, Ignacio Ibáñez Fernández y Borja Romero Martín \\ Universidad de La Laguna
}

RESUMEN

El propósito de este estudio fue examinar la relación entre los Cinco Grandes dominios de la personalidad y el funcionamiento sexual, medido con el Multidimensional Sexuality Questionnaire. Se analizan los datos de 165 sujetos. El 58\% de ellos eran mujeres $(n=96)$ y 69 hombres. La edad media era de 26,9 años $(D T=8,18)$. Los resultados señalan que neuroticismo y extraversión son las dimensiones de personalidad más relevantes en relación con las actitudes sexuales. La extraversión aparece relacionada con una perspectiva positiva de la sexualidad, mientras que el neuroticismo, se relaciona con una negativa. En cuanto a los factores sexuales, las diferencias se centran en estima, preocupación, motivación, ansiedad y control sexual externo, con puntuaciones superiores en hombres que en mujeres. En general, las relaciones entre personalidad y los factores sexuales, parecen estar moduladas por el género. Estos resultados muestran la importancia del estudio de las relaciones entre personalidad y funcionamiento sexual, atendiendo a las diferencias que puedan surgir en función del género, e invitan a continuar profundizando en este fenómeno.

Palabras clave: NEO-PI-R, Multidimensional Sexuality Questionnaire, funcionamiento sexual, diferencias de género, estudio ex post facto.

\section{ABSTRACT}

The purpose of this study was to examine the relationship between the Big Five factors of Personality and sexual factors (Multidimensional Sexuality Questionnaire). Data from 165 subjects were analysed, $58 \%$ of them were women ( $n=$ 96) and 69 men. With a mean age of 26.9 (SD $=8.18$ ). The results obtained show that, the personality dimensions that seem to be most relevant in connection with sexual attitudes, are Neuroticism and Extravertion. Overall, the Extravertion appears related to a positive perspective of sexuality, while the Neuroticism relates to a negative view. With regard to the sexual factors, differences focus on esteem, concern, motivation, anxiety and external sexual control, with higher scores in men than in women. In general, relationships between personality and sexual factors, seems to be modulated by sex. These results show the importance of the study of the relationships between personality factors and sexual attitudes, taking into account sexual differences, and require a deeper analysis of this phenomenon.

Keywords: NEO-PI-R, Multidimensional Sexuality Questionnaire, sexual functioning, sex differences, ex post facto study. 
Desde hace algunas décadas se ha ido prestando atención creciente al estudio de características psicológicas que influyen en las relaciones sexuales de las personas. En general, el análisis desde la psicología, abarca una temática diversa y, en muchos casos, centrada en aspectos concretos como los que aparecen más abajo.

Algunos trabajos aparecen relacionados con la ansiedad sexual (Janda \& O'Grady, 1980), las actitudes sexuales (Fisher \& Hall, 1988; Hendrick \& Hendrick, 1987; Lameiras \& Rodríguez, 2003; Ortega, Ojeda, Sutil, \& Sierra, 2005) y la estima sexual (Snell \& Papini, 1989). Además del análisis de diferencias entre géneros al elegir pareja (Eastwick \& Finkel, 2008; Wilbur \& Campbell, 2010), en las preferencias sexuales (Wilbur \& Campbell, 2010), el impacto de la personalidad en los sentimientos, pensamientos y conductas sexuales (Snyder, 1974, 1979; Snyder, Simpson, \& Gangestad, 1986) o, incluso, las actitudes sexuales positivas y negativas y su relación con el género (Macapagal \& Janssen 2011).

Otros estudios analizan el papel del bienestar psicológico o de la satisfacción en el funcionamiento sexual (Carrobles, Gámez-Guadixm \& Almendros, 2011), o la satisfacción sexual, especialmente en la mujer (Brody, 2007; Sills et al., 2005; Walker, 2006).

La investigación en la asertividad se remonta a la década de los setenta. Distintos estudios consideran que la asertividad sexual representa un componente central de la sexualidad humana (Stoner et al., 2008). Por lo cual, en la bibliografía al uso, se pueden encontrar un amplio número de ellos, con el análisis de la asertividad sexual en muestras de diferentes características (Caruthers, 2005; Greene \& Faulkner, 2005; Jacobs \& Thomlison, 2009; Mènard \& Offman, 2009; Oattes \& Offman, 2007). Además, la asertividad sexual aparece relacionada con diversos aspectos de la respuesta sexual, como el deseo y la satisfacción sexual (Santos-Iglesias \& Sierra, 2010).

Una temática que ha sido centro de nuestro interés, por su papel destacado en la sexualidad humana, es la importancia de las actitudes y conductas dentro de las relaciones sexuales. La manifestación de unas actitudes sexuales positivas suelen ir aparejadas con una mayor satisfacción sexual (Trudel, 2002).

Son muchos los estudios que han analizado la existencia de diferencias entre mujeres y hombres en distintos aspectos sexuales (Fink, Brewer, Fehl, \& Neave, 2007; Erol \& Orht, 2011; Kuhle, 2011; Pierce \& Hurlbert, 1999; Stulhofer, Graham, Bozievic, Kufrin, \& Ajdukovic, 2007), aunque los resultados son distintos. Mientras que, por ejemplo, para los últimos autores (Stulhofer et al., 2007), las mujeres muestran más asertividad sexual que los hombres, para otros (Pierce \& Hurlbert, 1999), el resultado es el contrario.

En general el interés de los personólogos por la sexualidad ha sido escaso, aunque un amplio número de autores defienden la importancia de la personalidad sobre el funcionamiento sexual y en concreto sobre las actitudes sexuales, (Bourdage, Lee, Ashton, y Perry, 2007; Heaven, Fitzpatrick, Craig, Kelly, \& Sebar, 2000; Heaven et al., 2003; Miller et al., 2004), pese a la debilidad e inconsistencia de los resultados (Smith, Nezlek, Webster, \& Paddock, 2007). 
Históricamente los primeros trabajos surgen de la mano del equipo de Eysenck (Eysenck, 1972, 1976; Eysenck \& Wilson, 1979), a partir del análisis de las relaciones entre personalidad y distintos aspectos y actitudes sexuales. Este autor recoge dos tipos de actitudes sexuales. Por una parte, actitudes frente a prácticas o actividades concretas (homosexualidad, frecuencia de uso del preservativo, etc.). Por otra, actitudes hacia la sexualidad como un todo, que puede conllevar satisfacción, miedos sexuales, ansiedad sexual, etc. Este último tipo, de carácter más psicológico, es cercano al interés que mueve el presente estudio.

Estos trabajos de Eysenck condujeron a importantes conclusiones dentro de las relaciones entre personalidad y aspectos sexuales como las actitudes sexuales. Como la tendencia de los extravertidos, en comparación con los introvertidos, a tener más parejas sexuales, a experimentar con distintas actividades sexuales y a tener sexo con más frecuencia, además de mayores niveles de satisfacción sexual. O la tendencia de las personas con puntuaciones altas en Neuroticismo a puntuar alto en ansiedad, preocupación, a ser más temerosas, y a ser más probable que muestren nerviosismo sexual, altos niveles de inhibición sexual y niveles más bajos de satisfacción sexual, en comparación con los que puntúan bajo.

Hoyle, Fejfar y Miller (2000), cuestionan el uso de modelos de personalidad psicobiológicos y han ignorado modelos taxonómicos como el Modelo de los Cinco Grandes (Five Factor Model, FFM, Costa \& McCrae, 1992).

Entre las investigaciones que emplean este modelo para el estudio de distintos aspectos de la sexualidad, y en relación con las actitudes sexuales, destacan las llevadas a cabo por autores como Snell (Snell, Fisher, \& Miller, 1991; Snell, Fisher, \& Walters, 1993) y Heaven (Heaven et al., 2000; Heaven et al., 2003). Estos últimos analizan las actitudes sexuales a través del Multidimensional Sexuality Questionnaire de Snell et al. (1993).

En las investigaciones del grupo de Snell se encontraron, en los hombres, mayores niveles en las variables sexuales de estima, preocupación, motivación, asertividad, y control sexual externo, que en las mujeres. Según Heaven (Heaven et al., 2003; Heaven et al., 2000) los hombres puntúan significativamente más alto que las mujeres en medidas relacionadas con la curiosidad y con la excitación sexuales y éstas más en satisfacción sexual.

Respecto a las relaciones significativas entre personalidad y los factores de actitudes hacia el sexo del estudio de Snell et al. (1993), surgieron relaciones negativas y significativas de Responsabilidad y Cordialidad con la preocupación sexual, y de Cordialidad con la motivación sexual. Heaven et al. (2000), informan que éstas se dan fundamentalmente entre Neuroticismo ( $\mathrm{N}$ ) y ciertos factores sexuales (con culpa e insatisfacción sexual en mujeres; y con culpa, excitación y curiosidad sexual, en hombres). Entre las personas con puntuaciones altas en Neuroticismo parece haber poca evidencia de satisfacción sexual o de habilidad sexual. En Extraversión (E), las relaciones significativas se dieron únicamente en mujeres (con curiosidad y excitación sexuales). El resto de dimensiones de la personalidad parecen mantener escasas relaciones con las actitudes sexuales (con 
Apertura a la experiencia y Responsabilidad), o prácticamente nulas (en Cordialidad). En general, los predictores más importantes de las actitudes sexuales son $\mathrm{N}$ y $\mathrm{E}$, con Responsabilidad y Apertura a la experiencia, en menor grado. Heaven et al. (2003), confirma la relevancia de E y $\mathrm{N}$ y el menor peso de Responsabilidad.

Algunos aspectos quedan aún por aclarar si se analizan estudios como el de Miller et al. (2004). Estos autores también defienden la importancia de la personalidad en el estudio de la conducta sexual. Aunque entre sus resultados señalan que surge cierta ambigüedad en la relación entre afecto negativo (neuroticismo de Eysenck), o emocionalidad negativa (Tellegen, 1985) y conductas sexuales de riesgo (en ambos casos con ausencia de relaciones significativas). Por el contrario, otros estudios (Hoyle, et al., 2000) han encontrado relación entre neuroticismo-ansiedad de Zuckerman y neuroticismo (del FFM) y algunas formas de riesgo sexual. Miller et al. (2004), sugieren que cuando se consideran a la vez otras dimensiones de personalidad, éstas no representan una contribución importante. Los resultados obtenidos hasta el momento son, además de escasos y dispares (Heaven et al., 2000; Heaven et al., 2003), lo que justificaría su estudio.

Los estudios que relacionan la personalidad con distintos aspectos de la sexualidad humana en adultos, como las actitudes y las conductas sexuales son claramente escasos, y esta cuestión no se ha resuelto en los últimos años. Por ello, como objetivo general del trabajo, se plantea continuar la línea de investigaciones anteriores en este campo (Eysenck, 1976; Heaven et al. 2000; Heaven et al., 2003). Para ello examinamos los vínculos existentes entre la percepción que tiene la persona acerca de su funcionamiento sexual y el FFM aportando la variable diferencias sexuales de la muestra, a este análisis.

Como hipótesis de trabajo se plantearon las siguientes:

1) Las dimensiones de personalidad extraversión y neuroticismo mantendrán, relaciones significativas con distintos factores sexuales (Eysenck, 1972, 1976; Eysenck \& Wilson, 1979; Costa \& McCrae, 1985; Heaven et al., 2000). Mientras que las relaciones con Responsabilidad, Apertura a la experiencia y Cordialidad, serán puntuales (Heaven et al., 2000; Heaven et al., 2003).

2) Los predictores más importantes de los distintos factores sexuales serán Extraversión y Neuroticismo (Heaven et al., 2000).

3) En cuanto a las diferencias de género, los hombres y las mujeres mostrarán un patrón diferencial en la relación entre personalidad y sexualidad (Feingold, 1992; Heaven et al., 2000): (a) los hombres obtendrán puntuaciones mayores que las mujeres en los factores sexuales de estima, preocupación, motivación, asertividad, y control externo (Snell et al., 1991; Snell et al., 1993); y (b) las mujeres mostrarán puntuaciones más altas en satisfacción sexual que los hombres (Eysenck, 1976; Heaven et al., 2000).

\section{Método}

\section{Participantes}

Un total de 165 personas completaron los cuestionarios. El 58\% de ellos eran mujeres ( $n$ =96) y 69 hombres. La edad media era de 26,9 
años $(D T=8,18)$, y la moda de 24 . La mayoría de ellos, 87 eran estudiantes (53\%) y el resto, 78 trabajadores (la totalidad con estudios de Bachillerato Unificado Polivalente, Formación Profesional, o superiores).

La participación fue voluntaria, anónima e individualizada, garantizando la confidencialidad de sus datos, previo consentimiento informado expreso.

\section{Instrumentos}

Multidimensional Sexuality Questionnaire (MSQ). (Snell et al., 1991 ; Snell et al., 1993). Este cuestionario consta de 60 ítems con una escala de respuesta tipo Likert de 5 puntos. A partir de esta prueba surge 12 factores (con 5 ítems cada uno), que miden la percepción que tienen las personas sobre distintos aspectos de su funcionamiento sexual (Heaven et al., 2003): estima sexual (alfa de Cronbach $=0,87$ ), preocupación sexual (alfa $=0,94$ ), control sexual interno (alfa $=0,80$ ), conciencia sexual (alfa $=$ $0,71)$, motivación sexual (alfa $=0,91$ ), ansiedad sexual (alfa $=0,83$ ), asertividad sexual (alfa $=$ $0,77)$, depresión sexual (alfa $=0,92$ ), control sexual externo (alfa $=0,86$ ), control social sexual (alfa $=0,90)$, miedo a las relaciones sexuales (alfa $=0,82)$, y satisfacción sexual (alfa $=0,94)$. Las escalas muestran validez y fiabilidad adecuadas (Snell et al., 1991; Snell et al., 1993).

NEO-PI-R. (Costa \& McCrae, 1992), para evaluar dimensiones normales de personalidad, adaptado al español por TEA Ediciones (1999). Compuesto por 240 ítems, mide cinco dimensiones (con 48 ítems cada una): Extraversión (alfa de Cronbach $=0,89$ ), Neuroticismo (alfa $=0,92)$, Apertura a la experiencia (alfa $=0,87$ ),
Cordialidad (alfa $=0,86$ ), y Responsabilidad (alfa $=0,90), y$ evalúa seis facetas dentro de cada una de ellas. Se han llevado a cabo muchos estudios con el cuestionario y ha mostrado de forma sistemática, buena fiabilidad y validez (Costa, McCrae, \& Dye, 1991).

\section{Procedimiento}

Se realizó un estudio descriptivo-correlacional con un diseño ex post facto transversal. Se empleó una muestra incidental de participantes voluntarios, obtenida siguiendo la técnica de muestreo de bola de nieve (muestreo no probabilístico), un total de 18 alumnos de $3^{\circ}$ de psicología formaron parte del programa de prácticas voluntarias con el fin de desempeñar el papel de evaluadores. Para ello fueron entrenados en la administración de las distintas pruebas e instruidos para que seleccionaran de su entorno a un grupo de entre ocho a diez personas adultas, distribuidas homogéneamente por género y edad. Una vez que los evaluadores escogieron a esa muestra, se les informó del carácter experimental del trabajo y se les pidió su participación voluntaria cumplimentando los distintos cuestionarios e inventarios. Se pidió el consentimiento informado y un teléfono de contacto. Las pruebas se cumplimentaban en unos 50 minutos.

Los datos obtenidos fueron analizados empleando el paquete estadístico SPSS para Windows en su versión 20.

Se analizaron las relaciones entre factores sexuales y personalidad. Además se realizaron análisis por separado para hombres y mujeres.

1.- Para el estudio de las diferencias en factores sexuales entre hombres y mujeres se re- 
curren a ANOVA, siguiendo a Snell et al. (1991) y Snell et al. (1993) al presentar los datos de validación del MSQ.

2.- Con el objeto de ver la capacidad explicativa de los Cinco Grandes de la personalidad sobre los distintos aspectos sexuales, se realizaron análisis de regresión lineal múltiple. Con el método paso a paso. Éste es un método usual, ha sido específicamente empleado en estudios similares y permite introducir de forma sucesiva las variables que tienen una contribución más importante para explicar la variable dependiente (Howitt \& Cramer, 2011).

3.- El análisis de regresión se realizó para hombres y mujeres, independientemente.

En todos los casos se analizaron las puntuaciones directas, sin ningún tipo de transformación.

\section{Resultados}

Se llevó a cabo un análisis correlacional de las relaciones entre personalidad y el funcionamiento sexual, y se realizó de forma independiente para hombres y mujeres (Tabla 1).

El análisis general de los resultados entre las distintas factores sexuales y las dimensiones de personalidad básicas, permite observar diferencias considerables en los patrones correlacionales seguidos por ambos géneros.

Al analizar estos datos, se encuentra que las relaciones tienen un mayor peso en los hombres, con un total de 31 correlaciones significativas (frente a 23 en las mujeres). Las dimensiones de personalidad más relevantes (en ambos géneros) son Extraversión (E) y Neuroticismo (N).

Los resultados son diferentes para cada género. Respecto a Extraversión, su papel se re- laciona más con lo que podríamos llamar una concepción positiva de la sexualidad. Así, en ambos sexos se mantienen relaciones positivas con estima, conciencia sexual, control interno, asertividad y satisfacción. En mujeres aparecen también relaciones positivas con motivación. En los hombres, encontramos relaciones negativas entre Extraversión y las actitudes sexuales de ansiedad, depresión, control social y control externo.

En cuanto al Neuroticismo, en los hombres se relaciona más con una vivencia negativa de lo sexual (relaciones positivas con preocupación, control social, miedo, insatisfacción, locus de control externo $y$, en mayor grado, ansiedad y depresión). En las mujeres esta vivencia negativa es algo más tenue, y se da con estima, asertividad y satisfacción. La relación es positiva con ansiedad y depresión.

La dimensión de Responsabilidad (C), juega un papel más relevante en la muestra femenina, con relaciones negativas con preocupación y control externo, y positivas con conciencia, miedo y control interno. Para los hombres todas las relaciones son negativas y se limitan a preocupación, ansiedad y control social.

Un patrón diferente sigue la Apertura a la experiencia $(\mathrm{O})$, que mantiene correlaciones positivas con los factores sexuales, especialmente en las mujeres (si bien de escasa cuantía). Mientras que en ambas muestras las relaciones se mantienen con estima, conciencia y control interno, se relaciona únicamente con motivación, asertividad y satisfacción en las mujeres.

Respecto al factor de Cordialidad (A), todas las relaciones son negativas. Con un patrón 
Tabla 1

Correlaciones entre personalidad y factores de funcionamiento sexual por género

\begin{tabular}{|c|c|c|c|c|c|c|c|c|c|c|}
\hline \multirow{3}{*}{$\begin{array}{l}\text { Factores } \\
\text { sexuales }\end{array}$} & \multicolumn{10}{|c|}{ Personalidad } \\
\hline & \multicolumn{2}{|c|}{ Neuroticismo } & \multicolumn{2}{|c|}{ Extraversión } & \multicolumn{2}{|c|}{ Apertura } & \multicolumn{2}{|c|}{ Cordialidad } & \multicolumn{2}{|c|}{ Responsabilidad } \\
\hline & Hombres & Mujeres & Hombres & Mujeres & Hombres & Mujeres & Hombres & Mujeres & Hombres & Mujeres \\
\hline Estima & -.15 & $-.23^{*}$ & $.32^{* *}$ & $.33^{* *}$ & $.31^{* *}$ & $.40^{* * *}$ & $-.21^{*}$ & .03 & -.14 & .07 \\
\hline Preocupación & $.19^{*}$ & .10 & -.14 & -.09 & .00 & .06 & $-.45^{* * *}$ & $-.26^{*}$ & $-.36^{* * *}$ & $-.25^{*}$ \\
\hline Conciencia & -.04 & -.03 & $.26^{*}$ & $.42^{* * *}$ & $.21^{*}$ & $.45^{* * *}$ & $-.22^{*}$ & .08 & -.04 & $.22^{*}$ \\
\hline Motivación & -.07 & -.10 & .18 & $.27^{* *}$ & .10 & $.31^{* *}$ & $-.30^{* *}$ & -.07 & -.08 & -.09 \\
\hline Ansiedad & $.41^{* * *}$ & $.32^{* *}$ & $-.23^{*}$ & -.03 & -.04 & .04 & $-.32^{* *}$ & -.12 & $-.21^{*}$ & -.15 \\
\hline Asertividad & -.12 & $-.28^{* *}$ & $.26^{*}$ & $.34^{* *}$ & .05 & $.41^{* * *}$ & $-.34^{* *}$ & -.01 & -.03 & .05 \\
\hline Depresión & $.42^{* *}$ & $.23^{*}$ & $-.38^{* * *}$ & -.15 & -.05 & -.06 & $-.19^{*}$ & -.05 & -.16 & -.13 \\
\hline Control social & $.34^{* *}$ & .16 & $-.28^{* *}$ & .10 & -.11 & -.02 & -.18 & -.18 & -.08 & -.05 \\
\hline Miedo & $.35^{* *}$ & .17 & -.17 & .02 & .06 & -.04 & .16 & -.14 & $-.19 *$ & -.11 \\
\hline Satisfacción & $-.33^{* *}$ & $-.25^{*}$ & $.42^{* * *}$ & $.24^{*}$ & .08 & $.19^{*}$ & .13 & .13 & .16 & .18 \\
\hline Control interno & .00 & -.12 & $.24^{*}$ & $.38^{* * *}$ & $.29^{* *}$ & $.35^{* * *}$ & $-.20^{*}$ & .09 & -.08 & $.25^{*}$ \\
\hline Control externo & $.36^{* *}$ & $-.25^{*}$ & .05 & .06 & -.04 & .05 & -.26 & -.07 & -.17 & $-.31^{* *}$ \\
\hline
\end{tabular}

Nota: ${ }^{*}=p<0,05 ;{ }^{* *}=p<0,01 ;{ }^{* * *}=p<0,001$.

claramente diferenciado en ambas muestras. En la masculina, se relacionan con casi la totalidad de los aspectos sexuales, mientras que en la femenina únicamente con preocupación.

Si nos centramos en los factores sexuales, las diferencias entre ambos sexos parecen encontrarse en los factores de control social sexual, ansiedad, depresión y control sexual interno. En todos aparecen mayor número de relaciones significativas en el caso masculino. El factor de asertividad sexual parece tener un mayor peso en la muestra femenina.

Finalmente, los factores sexuales que parecen mantenerse más independientes de personalidad en ambas muestras son: el miedo a las relaciones sexuales y la motivación sexual (Tabla 2).
Se analizaron las diferencias de género (Anova) en personalidad y funcionamiento sexual. Respecto a las primeras, se limitan a Cordialidad ( $F=20,39, p<0.001)$, con puntuaciones significativamente mayores para las mujeres. En cuanto a los distintos aspectos sexuales analizados (Tabla 2), los hombres puntúan más alto que las mujeres en la casi totalidad de factores, con la excepción de satisfacción sexual y control sexual interno; aunque con significación sólo para estima, preocupación, motivación, ansiedad y control sexual externo.

Por último, se realizaron diferentes análisis de regresión lineal múltiple, con la intención de determinar la capacidad explicativa de la personalidad sobre la percepción acerca del funcionamiento sexual propio. Se optó por el 
Tabla 2

Medias y desviaciones típicas (D.T.) en factores sexuales en mujeres y hombres

\begin{tabular}{|c|c|c|c|c|c|}
\hline \multirow{2}{*}{ Factores sexuales } & \multicolumn{2}{|c|}{ Hombres $(N=69)$} & \multicolumn{2}{|c|}{ Mujeres $(N=96)$} & \multirow[b]{2}{*}{$F$} \\
\hline & Media & $D T$ & Media & $D T$ & \\
\hline Estima sexual & 12.28 & 3.98 & 10.77 & 3.92 & $5.81^{* *}$ \\
\hline Preocupación sexual & 8.23 & 2.79 & 5.95 & 1.95 & $37.88^{* * *}$ \\
\hline Conciencia sexual & 13.30 & 3.20 & 13.19 & 2.80 & 0.06 \\
\hline Motivación sexual & 13.12 & 4.15 & 10.36 & 4.33 & $16.67^{* * *}$ \\
\hline Ansiedad sexual & 6.29 & 3.67 & 5.12 & 3.36 & $4.49^{*}$ \\
\hline Asertividad sexual & 11.83 & 3.82 & 11.43 & 4.19 & 0.39 \\
\hline Depresión sexual & 4.28 & 5.15 & 3.01 & 4.58 & 2.73 \\
\hline Control social sexual & 3.41 & 4.10 & 2.68 & 3.29 & 1.57 \\
\hline Miedo a relaciones sexuales & 6.86 & 3.41 & 6.12 & 2.96 & 2.17 \\
\hline Satisfacción sexual & 11.75 & 5.70 & 12.82 & 5.34 & 1.50 \\
\hline Control sexual interno & 12.68 & 3.67 & 12.74 & 3.67 & 0.01 \\
\hline Control sexual externo & 4.59 & 5.00 & 3.15 & 3.48 & $4.73^{*}$ \\
\hline
\end{tabular}

Nota. $D T=$ desviación típica. ${ }^{*}=p<0.05 ;^{* *}=p<0.01 ;{ }^{* * *}=p<0.001$.

método paso a paso y por analizar los hombres y las mujeres de forma independiente. Los principales resultados se encuentran en la Tabla 3.

En general, y como en el análisis correlacional, Extraversión aparece relacionada con una perspectiva positiva de la sexualidad (conciencia, motivación, asertividad, satisfacción y control interno), independientemente del género. Mientras que el Neuroticismo tendría que ver con un vivencia negativa (ansiedad, asertividad, depresión, control social, miedo a las relaciones sexuales y satisfacción).

Además, en el caso del Neuroticismo, esta relación negativa con la sexualidad sería distinta para cada sexo: Marcada por ansiedad, control social sexual, miedo a las relaciones se- xuales y control sexual externo para los hombres y baja asertividad para las mujeres. La depresión sería común a ambos.

La Responsabilidad se relaciona negativamente con la depresión en hombres. En mujeres, positivamente con el control interno y negativamente con el control externo sexual.

Mientras la Apertura a la experiencia parece mas relevante para explicar las actitudes sexuales femeninas y, Cordialidad, las masculinas.

\section{Discusión}

La finalidad de este estudio fue examinar las relaciones entre personalidad (en base al FFM) y la percepción que tienen las personas de sus relaciones sexuales. Los resultados obtenidos, muestran con cierta claridad cómo 
Tabla 3

Análisis de regresión de las variables de personalidad sobre los factores sexuales, por género

\begin{tabular}{|c|c|c|c|c|c|c|c|c|c|}
\hline \multirow{3}{*}{ Estima sex } & & \multicolumn{4}{|c|}{ Hombres $(N=69)$} & \multicolumn{4}{|c|}{ Mujeres $(N=96)$} \\
\hline & & \multirow[t]{2}{*}{$F$} & \multirow[t]{2}{*}{$\beta$} & \multirow[t]{2}{*}{$t$} & \multirow[t]{2}{*}{$\mathrm{R}^{2}$} & \multirow[t]{2}{*}{$F$} & \multirow[t]{2}{*}{$\beta$} & \multirow[t]{2}{*}{$t$} & \multirow[t]{2}{*}{$\mathrm{R}^{2}$} \\
\hline & & & & & & & & & \\
\hline & $E$ & & .37 & $3.29^{* * *}$ & & & & & \\
\hline & $\mathrm{O}$ & & & & & & .40 & $4.13^{* * *}$ & \\
\hline & A & $7.23^{* * *}$ & -.28 & $-2.45^{*}$ & .18 & $.17^{* * *}$ & & & .16 \\
\hline \multicolumn{10}{|c|}{ Preocupación } \\
\hline & A & & -.39 & $-3.59^{* * *}$ & & & -.26 & $-2.57^{*}$ & \\
\hline & $\mathrm{C}$ & $12.38^{* * *}$ & -.28 & $-2.60^{* *}$ & .27 & $6.6^{*}$ & & & .07 \\
\hline \multicolumn{10}{|l|}{ Consciencia } \\
\hline & A & & -.27 & $-2.31^{*}$ & & & & & \\
\hline & $E$ & & .31 & $2.62^{* *}$ & & & .26 & $2.22^{*}$ & \\
\hline & $\mathrm{O}$ & $5.21^{* *}$ & & & .14 & $15.96^{* * *}$ & .30 & $2.50^{*}$ & .26 \\
\hline \multicolumn{10}{|l|}{ Motivación } \\
\hline & $\mathrm{O}$ & & & & & & .32 & $3.19^{* *}$ & \\
\hline & $A$ & & -.35 & $-3.00^{* *}$ & & & & & \\
\hline & $E$ & $5.75^{* *}$ & .24 & $2.08^{*}$ & .15 & $10.14^{* *}$ & & & .10 \\
\hline \multicolumn{10}{|l|}{ Ansiedad } \\
\hline & $\mathrm{N}$ & & .41 & $3.70^{* * *}$ & & & & & \\
\hline & $A$ & $13.72^{* * *}$ & & & .17 & $9.06^{* *}$ & .30 & $3.01^{* *}$ & .09 \\
\hline \multicolumn{10}{|l|}{ Asertividad } \\
\hline & $\mathrm{O}$ & & & & & & .37 & $3.92^{* * *}$ & \\
\hline & $E$ & & .33 & $2.95^{* *}$ & & & & & \\
\hline & $A$ & & -.40 & $-3.62^{* * *}$ & & & & & \\
\hline & $\mathrm{N}$ & $9.32^{* * *}$ & & & .22 & $12.19^{* * *}$ & -.21 & $-2.26^{*}$ & .21 \\
\hline \multicolumn{10}{|l|}{ Depresión } \\
\hline & $\mathrm{N}$ & $14.63^{* * *}$ & .42 & $3.83^{* * *}$ & .18 & $4.03^{*}$ & .21 & $2.01^{*}$ & .04 \\
\hline \multicolumn{10}{|l|}{ Contr. social } \\
\hline & $\mathrm{N}$ & $8.94^{* *}$ & .34 & $2.99^{* *}$ & .12 & --- & --- & --- & --- \\
\hline \multicolumn{10}{|l|}{ Miedo } \\
\hline & $A$ & & .28 & $2.40^{*}$ & & & & & \\
\hline & $\mathrm{N}$ & $7.78^{* * *}$ & .42 & $3.67^{* * *}$ & .19 & --- & --- & --- & --- \\
\hline \multicolumn{10}{|l|}{ Satisfacción } \\
\hline & $E$ & $13.96^{* * *}$ & .42 & $3.74^{* * *}$ & & & .25 & $2.47^{*}$ & \\
\hline \multicolumn{10}{|l|}{ Control-I } \\
\hline & $\mathrm{C}$ & & & & & & .20 & $2.12^{*}$ & \\
\hline & $\mathrm{O}$ & & .29 & $2.43^{*}$ & & & & & \\
\hline & $E$ & $5.92^{*}$ & & & .08 & $10.99^{* * *}$ & .36 & $3.83^{* * *}$ & .19 \\
\hline \multicolumn{10}{|l|}{ Control-E } \\
\hline & $\mathrm{N}$ & & .36 & $3.12^{* *}$ & & & & & \\
\hline & $\mathrm{C}$ & $9.72^{* *}$ & & & & & -.31 & $-3.08^{* *}$ & \\
\hline
\end{tabular}

Nota. Se presenta el valor de $F$ y la varianza explicada $\left(\mathrm{R}^{2}\right)$ para el modelo en su conjunto y los valores de t y $\beta$ para cada variable del modelo; $\mathrm{E}=$ Extraversión, $\mathrm{N}=$ Neuroticismo, $\mathrm{O}=$ Apertura a la experiencia, $\mathrm{A}=$ Cordialidad y $C=$ Responsabilidad. ${ }^{*}=p>0,05 ;{ }^{* *}=p>0,01 ;{ }^{* *}=p>0,001$. 
algunas dimensiones de personalidad están relacionadas con el funcionamiento sexual, si bien existen diferencias importantes entre hombres y mujeres.

Las relaciones positivas obtenidas en diferentes ocasiones entre Extraversión y distintos factores sexuales (Eysenck, 1972, 1976; Eysenck \& Wilson, 1979), constituyen uno de los resultados de peso que se replicaron en este trabajo. En esta línea, los datos parecen indicar que, tanto en hombres como en mujeres, existe una relación estrecha y negativa del Neuroticismo con el funcionamiento sexual (y en general con lo que se ha denominado una vivencia negativa de la sexualidad), aquí podemos destacar el papel de la satisfacción en la dirección esperada. Esto también se confirma exclusivamente en la muestra masculina, en los factores sexuales de preocupación y miedo.

De acuerdo con Hoyle et al. (2000), que encuentran la Responsabilidad, prácticamente independiente de los distintos factores sexuales, en el presente trabajo, el papel de la Responsabilidad parece ser poco significativo en el funcionamiento sexual. Con escasas relaciones con la mayoría de factores sexuales y relaciones negativas con preocupación sexual (Snell et al., 1993). En cuanto a Apertura a la experiencia, parece mantener ciertas relaciones con factores sexuales en mujeres, a la vez que parece tener un aceptable poder predictivo con esta muestra.

En general, los análisis sobre personalidad y sexualidad apoyan la primera hipótesis del estudio. Aunque los resultados indican una relación importante y un buen poder predictivo de la dimensión de personalidad Cordia- lidad con los factores sexuales exclusivamente en hombres. Esto apoyaría sólo la hipótesis 1 en la muestra femenina. Aunque se confirma la segunda parte de la hipótesis con los dos sexos, que señalaba que de haber relaciones se darán con preocupación sexual (Snell et al., 1993).

De acuerdo con los resultados de Hoyle et al. (2000), la investigación actual muestra que, Neuroticismo y Extraversión parecen tener un papel importante y diferente al de otros factores de personalidad, de cara a la predicción de las funciones sexuales (hipótesis 2).

Como se anticipó hay un número amplio de resultados que apoyan el patrón diferencial entre géneros planteado en la tercera hipótesis (Feingold, 1992; Heaven et al., 2000). Algunos de ellos se citarán a continuación:

En la muestra masculina la ansiedad sexual desempeña un peso considerable en su relación con la casi totalidad de dimensiones de personalidad. No ocurre lo mismo en la muestra femenina. Este factor sexual tiene un papel importante en la diferenciación entre ambos sexos.

Además, un dato sorprendente es que aunque los resultados obtenidos hasta el momento relacionan más la dimensión de Neuroticismo con ansiedad y depresión en mujeres, aquí Neuroticismo predice ansiedad únicamente en los hombres. Esto parece indicar que en el funcionamiento sexual, este factor está más asociado a los hombres.

Como ya hemos comentado, en mujeres la dimensión de Apertura a la experiencia se relaciona más con el funcionamiento sexual y en hombres la Cordialidad. 
Sobre el hallazgo de diferencias significativas en factores sexuales, a favor de la muestra masculina, los resultados las refrendan, con la excepción del factor de asertividad.

Por último, un resultado sorprendente, ya que iría en contra de lo esperado, es que en la muestra actual no surgen diferencias significativas en asertividad sexual entre ambas muestras, tal y como plantearon Snell et al. (1993).

En resumen, se puede apreciar un patrón diferencial de relaciones según que el funcionamiento sexual sea masculino o femenino, indicando que se observa una percepción del propio funcionamiento sexual que parece ser fundamentalmente masculina y otra femenina. En éste último caso, se hace patente el cambio social en cuanto a la evolución de los roles sociales, lo que se traduce en un mayor compromiso de las mujeres con una visión más positiva de la sexualidad y en el caso de los hombres, una visión más negativa del hecho sexual (mayor miedo, preocupación y ansiedad sexual) reforzando así el papel más activo de las mujeres.

En conclusión, en las relaciones entre los factores de personalidad y las percepciones sobre las tendencias sexuales, parecen tener un mayor peso las dimensiones de personalidad de Extraversión y Neuroticismo tanto en hombres como en mujeres, y con importantes aspectos comunes. Desde un punto de vista predictivo, Apertura a la experiencia y Cordialidad también parecen ser relevantes (con un mayor peso cuando analizamos cada sexo por separado). Aunque ambas muestras no arrojan diferencias significativas en dimensiones de personalidad (con la excepción de Cordia- lidad), surgen considerables diferencias en los factores sexuales.

Consideramos que este estudio aporta información de interés al referirse a una muestra española, que no incluye únicamente estudiantes universitarios. Además, ahonda en una temática sobre la que, incluso en la última década, existe escasa investigación. A pesar de ello, los resultados que se presentan deben ser vistos como tentativos y precisan replicación, a ser posible, tanto con muestras sociológicamente comparables como dispares y de mayor amplitud (para garantizar su generalización). A pesar de todo ello, no se puede olvidar que quedan pocas dudas sobre la existencia de relaciones complejas, sorprendentes y de interés creciente, entre los rasgos de personalidad y los diferentes aspectos de la sexualidad, lo que resulta alentador de cara a continuar investigando sobre el tema.

\section{Referencias}

Bourdage, J. S., Lee, K., Ashton, M. C., \& Perry, A. (2007). Big Five and HEXACO model personality correlates of sexuality. Personality and Individual Differences, 43, 1506-1516.

Brody, S. (2007). Intercourse orgasm consisten$c y$, concordance of women's genital and subjective sexual arousal, and erotic stimulus presentation sequence. Journal of Sex and Marital Therapy, 33, 31-39.

Carrobles, J. A., Gámez-Guadix, M., \& Almendros, C. (2011). Funcionamiento sexual, satisfacción sexual y bienestar psicológico y subjetivo en una muestra de mujeres españolas. Anales de Psicología, 27, 27-34.

Caruthers, A. S. (2005). "Hookups" and "Friends with benefits": Nonrelational sexual encounters as contexts of women's normative sexual development. Tesis doctoral. University of Michigan, Michigan.

Costa, P. T. \& McCrae, R. R. (1985). Manual of 
the NEO Personality Inventory: Form $S$ and Form R. Odessa, FL, EUA: Psychological Assessment Resources.

Costa, P. T., \& McCrae, R.R. (1992). Revised NEO Personality Inventory (NEO-PI-R) and NEO Five-Factor Inventory (NEO-FFI); professional manual. Odessa, FL, EUA.: Psychological Assessment Resources (Adaptación española, 1999, TEA Ediciones, S. A.).

Costa, P. T. , McCrae, R. R., \& Dye, D. A. (1991). Facet scales for agreeableness and conscientiousness: a revision of the NEO Personality Inventory. Personality and Individual Differences, 12, 887-898.

Eastwick, P. W., \& Finkel, E. J. (2008). Sex differences in mate preferences revisited: Do people know what the initially desire in a romantic partner? Journal of Personality and Social Psychology, 94, 245-264.

Erol, R. Y., \& Orth, U. (2011). Self-Esteem development from age 14 to 30: A longitudinal Study. Journal of Personality and Social Psychology, 3, 607-619.

Eysenck, H. J. (1972). Psychology is about people. Harmondsworth, Londres, Great Britain: Penguin.

Eysenck, H. J. (1976). Sex and personality. London: Open Books.

Eysenck, H. J., \&Wilson, B. (1979). The psychology of sex. London: Dent.

Feingold, A. (1992). Gender differences in mate selection preferences: A test of the parental investment model. Psychological Bulletin, 122, 125-139.

Fink, B., Brewer, G., Fehl, K., \& Neave, N. (2007). Instrumentality and lifetime number of sexual partners. Personality and Individual Differences, 43, 747-756.

Fisher, T. D., \& Hall, R. G. (1988). A scale for the comparison of the sexual attitudes of adolescents and their parents. Journal of Sex Research, 24, 90-100.

Greene, K., \& Faulkner, S.L. (2005). Gender, belief in the sexual double standard, and sexual task in heterosexual dating relationships. Sex Roles, 53, 239-251.

Heaven, P. C. L., Crocker, D., Edwards, B., Preston, N., Ward, R., \& Woodbridge, N. (2003).
Personality and sex. Personality and Individual Differences, 35, 411-419.

Heaven, P. C. L., Fitzpatrick, J., Craig, F. L., Kelly, P., \& Sebar, G. (2000). Five personality factors and sex: preliminary findings. Personality and Individual Differences, 28, 1133-1141.

Hendrick, S. S. \& Hendrick, C. (1987). Multidimensionality of sexual attitudes. Journal of Sex Research, 23, 502-526.

Howitt, D., \& Cramer, D. (2011). Introduction to SPSS Statistics in Psychology (5th ed.). Harlow: Pearson Education.

Hoyle, R. H., Fejfar, M. C., \& Miller, J. D. (2000). Personality and sexual risk taking: a quantitative review. Journal of Personality, 68, 1203-1231.

Jacobs, R. J., \& Thomlison, B. (2009). Self-silencing and age as risk factors for sexually acquired HIV in midlife and older women. Journal of Aging and Health, 21, 102-128.

Janda, L. H., \& O'Grady, K. E. (1980). Development of a sex anxiety inventory. Journal of Consulting and Clinical Psychology, 48, 169175.

Kuhle, B. X. (2011). Did you have sex with him? Do you love her? An in vivo test of sex differences in jealous interrogations. Personality and Individual Differences, 51, 1044-1047.

Lameiras, M., \& Rodríguez, Y. (2003) The Big Five and sexual attitudes in Spanish students. Social Behavior and Personality, 31, 357-362.

Macapagal, K. R., \& Janssen, E. (2011). The valence of sex automatic affective associations in erotophilia and erotophobia. Personality and Sex and Marital Therapy, 28, 229-249. Recuperado de http://dx.doi. org/10.1016\%2Fj.paid.2011.06.008

Mènard, A. D., \& Offman, A. (2009). The interrelationships between sexual self-esteem, sexual assertiveness and sexual satisfaction. Canadian Journal of Human Sexuality, 18, 35-45.

Miller, J. D., Lynam, D., Zimmerman, R. S., Logan, T. K., Leukefeld, C., \& Clayton, R. (2004). The utility of the Five Factor Model in understanding risky sexual behavior. Personality and Individual Differences, 36, 1611-1626.

Oattes, M. K., \& Offman, A. (2007). Global self- 
esteem and sexual self-esteem as predictors of sexual communication in intimate relationships. Canadian Journal of Human Sexuality, 16, 89-100.

Ortega, V., Ojeda, P., Sutil, F., \& Sierra, J. C. (2005). Culpabilidad sexual en adolescentes: estudio de algunos factores relacionados. Anales de Psicología, 21, 268-275.

Pierce, A. P., \& Hurlbert, D. F. (1999). Test-Retest reliability of the Hurlbert Index of Sexual Assertiveness. Perceptual and Motor Skills, 88, 31-34.

Santos-Iglesias, P., \& Sierra, J.C. (2010). El papel de la asertividad sexual en la conducta humana: una revision sistemática. International Journal of Clinical and Health Psychology, 10, 553-577.

Sills, T., Wunderlich, G., Pyke, R., Segraves, R. T., Leiblum, S., Clayton, A.,... Evans, K. (2005). The sexual interest and desire inventoyfemale (SIDI-F): Item response analyses of data from women diagnosed with hypoactive sexual desire disorder. Journal of Sexual Medicine, 2, 801-818.

Smith, C. V., Nezlek, J. B., Webster, G. D., \& Paddock, E. L. (2007). Relationships between daily sexual interactions and domainspecific and general models of personality traits. Journal of Social and Personality Relationships, 24, 497-515.

Snell, W. E., Fisher, T. D., \& Miller, R. S. (1991). Development of the sexual awareness questionnaire: components, reliability, and validity. Annals of Sex Research, 4, 65-92.

Snell, W. E., Fisher, T. D., \& Walters, A. S. (1993). The Multidimensional Sexuality Questionnaire: An objective self-report measure of psychological tendencies associated with human sexuality. Annals of Sex Research, 6 , 27-55.

Snell, W. E., Jr., \& Papini, D.R. (1989). The sexuality Scale: An instrument to measure sexualesteem, sexual depression, and sexualpreoccupation. Journal of Sex Research, 26, 256-263.

Snyder, M. (1974). Self-monitoring of expressive behaviour. Journal of Personality and Social Psychology, 30, 526-537.
Snyder, M. (1979). Sef-monitoring processes. En L. Berkowitz (Ed.), Advances in experimental social psychology (Vol. 12, pp. 85-128). New York: Academic Press.

Snyder, M., Simpson, J.A., \& Gangestad, S. (1986). Personality and sexual relations. Journal of Personality and Social Psychology, 51, 181-190.

Stoner, S. A., Norris, J., George, W. H., Morrison, D. M., Zawacki, T., Davis, K. C., \& Hessler, D. M. (2008). Women's condom use assertiveness and sexual risk-taking: Effects of alcohol intoxication and adult victimization. Addictive Behaviors, 33, 1167-1176.

Tellegen, A. (1985). Structures of mood and personality and their relevance to assessing anxiety, with an emphasis on self-report. En A. H. Tuma, \& J. D. Maser (Eds.), Anxiety and the anxiety disorders (pp. 681-706). Hillsdale, NJ, EUA: Erlbaum.

Walker, D. P. (2006). Impaired sexual assertiveness and consensual sexual activity as risk factors for sexual coerción in heterosexual college women. Tesis doctoral. Universidad de Miami, Ohio.

Wilbur, J., \& Campbell, L. (2010). What do women want? An interactionist account of women's mate preferences. Personality and Individual Differences, 49, 749-754. 
